\title{
Die DeGIR der Zukunft: Innovativ, virtuell und vernetzt
}

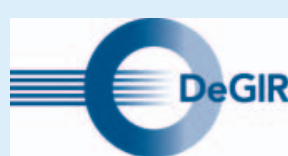

DEUTSCHE GESELLSCHAFT FÜR INTERVENTIONELLE RADIOLOGIE

UND MINIMAL-INVASIVE THERAPIE

in der Deutschen Röntgengesellschaft e.V.

Im Fokus seiner Amtszeit stehen die Begeisterung des interventionsradiologischen Nachwuchses und digitale Projekte: Prof. Dr. Marcus Katoh vom Helios Klinikum Krefeld ist seit Januar 2021 neuer Vorsitzender des Vorstands der DeGIR. Im Interview gibt er Einblick, welche Ziele er anvisiert und welche Themen ihm wichtig sind.

Herr Professor Katoh, am 13. Januar 2021 haben Sie das Amt des Vorsitzenden der
DeGIR übernommen - herzlichen Glückwunsch! Welche Ziele haben Sie sich für Ihre Amtszeit gesetzt?

Ein besonderes Anliegen ist mir die Begeisterung junger Ärztinnen und Ärzte für die Interventionelle Radiologie. Das ist einfacher gesagt als getan, aber in den letzten Jahren haben wir uns als Fachgesellschaft schon ziemlich gut aufgestellt und wichtige Anstöße gegeben. Ich habe das Glück, dass diese Anstöße zum Teil „nur noch“ fortgesetzt und intensiviert werden müssen.

Um bei den jungen Kolleginnen und Kollegen das Interesse zu wecken, aber auch, um Kolleginnen und Kollegen anderer Fachdisziplinen und Patientinnen und Patienten sowie die allgemeine Öffentlichkeit auf unsere Leistungen aufmerksam zu machen, wird zukünftig das bisherige Team Öffentlichkeitsarbeit zu einer Lenkungsgruppe. So wollen wir diese Arbeit noch enger mit der Arbeit im Vorstand der DeGIR verzahnen.

Was für die Akzeptanz unserer Leistungen innerhalb, insbesondere aber auch außerhalb der Radiologie ganz essentiell sein 


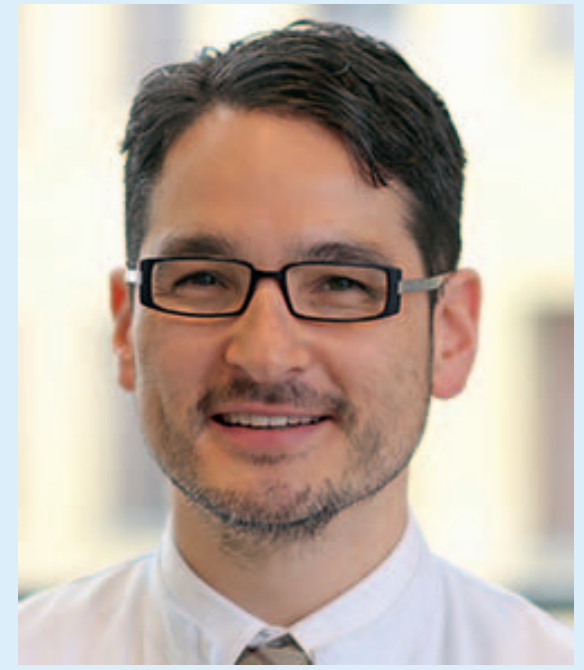

Prof. Dr. Marcus Katoh

wird, ist der Nachweis von umfassenden klinischen Kompetenzen bei der Versorgung unserer Patientinnen und Patienten. Diese werden mit darüber entscheiden, ob wir uns in Zukunft noch stärker als klinisches Fach und die interventionell-radiologischen Verfahren als integralen Bestandteil der Patientenversorgung etablieren können. Um auch hier Akzente zu setzen, wurde die Lenkungsgruppe „Klinische Integration" geschaffen.

Ein weiteres persönliches Anliegen ist mir die Weiterentwicklung unserer Simulationskurse. Diese sollen, nicht zuletzt durch die aktuelle Pandemie bedingt, in einem virtuellen Format umgesetzt werden.

Virtuelle Simulationskurse - das klingt nach einem anspruchsvollen Projekt. Wie dürfen wir uns so etwas vorstellen?

In den vergangenen Jahren haben wir es geschafft, moderne Präsenz-Simulationskurse mit hoch sensiblen elektronischen Simulatoren integriert in ein fesselndes Format aufzubauen, die ein sehr realitätsnahes Training erlauben.

Das Motto dieser Kurse lautete: „Mit den Augen klauen“. Sie können sich das so vorstellen: An einem Simulator arbeitet eine Expertin oder ein Experte, die simulierten Angiografiebilder werden in einer Großprojektion dargestellt, die Teilnehmenden, die auch jeweils an einem Simulator sitzen, gucken sich das erstmal an und führen dann Schritt für Schritt die gleichen Handgriffe am selben Fall durch. In diesen Kursen hatten wir auch die Möglichkeit, Gruppen aufzuteilen: Gruppe 1 löst das Problem mit einem Stent, Gruppe 2 mit dem Ballon et cetera. So ließen sich am selben Fall sogar unterschiedliche Resultate vergleichen.

Die virtuelle Variante ist ein Stück weit aus der Not geboren. Unsere ursprünglichen Ziele mussten wir zunächst vertagen: Wir wollten das 2020 eröffnete Röntgen-Geburtshaus nutzen und dort ein Simulationszentrum aufbauen. Während des Röntgenkongresses 2020 haben wir aber gesehen, welch' ein Erfolg aus digitalen Formaten wachsen kann. Nun hat natürlich die Digitalisierung eines Hands-on-Workshops viele weitere Dimensionen. Noch stecken wir mitten in der Vorbereitungsphase. Zum Testen haben wir in Krefeld und in München Simulatoren aufgebaut. Jedes dieser Geräte ist mit zwei Kameras ausgestattet. Die erste Kamera nimmt die Interventionalistin oder den Interventionalisten am Simulator auf, die zweite Kamera ist auf das Bedienumfeld gerichtet. Mit letzterer kann man die Aktivitäten während der Simulation und Materialien, wie zum Beispiel Stents, in Nahaufnahme zeigen. Diese Hardware kombinieren wir mit einem Online-Kommunikationsportal, mit dem die Standorte vernetzt werden. Wir waren selber erstaunt, wie gut das klappt. Wir können wahlweise nur die Simulationsbilder darstellen, oder aber einen Split-Screen mit Bildern der ersten und zweiten Kamera, die Bedienoberfläche des Simulators und dazu sogar noch die klinischen Daten einblenden. So haben die Teilnehmenden bzw. Zuhörenden die Möglichkeit, wie bei einem Präsenz-Simulationskurs alles Wichtige zu sehen und gleichzeitig am eigenen Simulator die Übungen direkt nachzuvollziehen.

Das bedeutet, ein Simulator vor Ort ist die notwendige Voraussetzung, um an einem solchen Kurs teilnehmen zu können?

Für die aktive Teilnahme: ja! Durch eine Kooperation mit der Firma Mentice haben wir vier weitere Simulatoren angemietet. Wir werden das beschriebene Konzept zunächst im kleinen Rahmen optimieren und dann ausweiten. Dazu werden wir Simulatoren an DeGIR-Ausbildungszentren verschicken, von denen aus die Lernenden aktiv an den Kursen teilnehmen können. Es wäre aber auch denkbar, dass weitere interessierte Kolleginnen und Kollegen als „passiv“ zugeschaltete Zuhörende an Interventionen und den entsprechenden Diskussionen teilnehmen.

An welche Zielgruppe richten sich die virtuellen Kurse - und können Sie schon verraten, wann der voraussichtliche Start ist?

Wahrscheinlich ist es erstmal einfacher, mit einem Anfänger-Angebot zu starten. Grundsätzlich ist aber alles denkbar: Komplikationsmanagementkurse, Master Classes beziehungsweise spezielle Interventionen für Fortgeschrittene.

Wenn die Optimierungen abgeschlossen sind und wir die ersten Zentren erfolgreich einbezogen haben, werden wir mit den ersten Kursen starten. Wir hoffen, dass das in Q2 oder Q3 2021 der Fall sein wird.

\section{Haben Sie denn ein ähnliches Konzept auch für den kommenden Röntgenkon- gress geplant?}

Das haben wir überlegt, ja. Die praktischen Übungen können, wie bereits erwähnt, nur an einem Simulator stattfinden. Aber wenn man einen simulierten Fall live verfolgbar macht und den Kurs z. B. mit Multiple Choice-Fragen unterfüttert, könnte ich mir vorstellen, dass das im Rahmen eines Kongresses sehr interessant sein könnte. Dadurch, dass wir keine echten Patientinnen und Patienten haben, können wir Kurse deutlich kompakter gestalten und viel Zeit für spezifische Fragen einräumen - in einem realen Behandlungsablauf wäre das nicht denkbar. Allerdings sind wir in Bezug auf dieses Konzept noch in den Anfängen.

Die digitalen Aktivitäten der DeGIR sollen nicht zuletzt auch ihre Entsprechung in einem aktualisierten Außenauftritt finden. Was genau haben Sie geplant?

Die Visitenkarte einer Fachgesellschaft und somit auch der DeGIR ist auch immer ihr Internetauftritt. Hier wollen wir etwas nachbessern! Wir wollen eine Webseite kreieren, die einladender und lebhafter ist. Gleichzeitig soll die Webseite ein Ort zur Vernetzung sein, gerade, wenn es darum geht, den Austausch zwischen befreundeten Gesellschaf- 
ten wie mit der europäischen Gesellschaft, der CIRSE, oder mit der Neuroradiologie, der DGNR, zu fördern und gegenseitig auf Programme und Aktivitäten aufmerksam zu machen. Außerdem haben wir viele spannende Projekte, beispielsweise Kolleginnen und Kollegen, die selbständig Apps entwickeln. Solchen Themen und aktuellen Projekten möchten wir eine angemessene Bühne geben.

Sie haben ja ganz zu Beginn unseres Gesprächs die Nachwuchsarbeit als eines der Ziele Ihrer Amtszeit benannt. Die DeGIR hat hier bereits Projekte auf den Weg gebracht, etwa die Kooptierung von Henrike-Renate Ziegler seitens des Forums Junge Radiologie in den DeGIR-Vorstand oder auch das Flinke Finger-Programm. Haben Sie noch weitere Ideen, wie die DeGIR junge Ärztinnen und Ärzte für die Interventionsradiologie und für die aktive Arbeit in der Fachgesellschaft gewinnen kann?
Durch die Kooptierung von Frau Ziegler aus dem Forum Junge Radiologie wird ein frischer Wind Einzug in die Diskussionen im Vorstand halten, da bin ich mir sicher. Das erlaubt uns Einblicke in die Erwartungen und Ansprüche der jüngeren Kolleginnen und Kollegen. Neue Kolleginnen und Kollegen sollen auch mit in der Arbeit der Lenkungsgruppen eine tragende Rolle übernehmen. Künftig wollen wir zudem Sektionen für bestimmte Krankheitsbilder etablieren; auch hier werden wir auf die Mitarbeit engagierter Kolleginnen und Kollegen angewiesen sein. Gerade was den Nachwuchs anbelangt, bin ich davon überzeugt, dass wir einen großen Vorteil gegenüber anderen Fachdisziplinen haben, den wir unbedingt nutzen müssen. Und wie aktiv dieser Nachwuchs ist, zeigt uns ja das Forum Junge Radiologie, das mittlerweile rund 2000 Mitglieder zählt. Wenn wir diese Kolleginnen und Kollegen zur Mitarbeit überzeugen wollen, dann ist das wie mit wissenschaftlichen Arbeiten: Man muss sie in konkrete Frage- stellungen mit klaren Zielen einbinden und dann laufen lassen.

Gibt es darüber hinaus noch Themen und Projekte, die Sie während Ihrer Amtszeit weiter vorantreiben möchten?

Das breite Spektrum in der Diagnostik und ihr zunehmender Einfluss bei therapeutischen Eingriffen machen den Reiz der Radiologie aus und sind zugleich aber auch ihre Angriffsfläche. Diese Flanken gilt es in enger Abstimmung mit der DRG, und den assoziierten AGs und Gesellschaften zu schützen und zu stärken. Auch hier wurden schon viele wichtige Weichen gestellt. Die Vergangenheit hat aber gezeigt, dass wir stets auf der Hut sein müssen, und auch auf die politischen Entwicklungen und Veränderungen schnell und adäquat reagieren müssen, damit wir noch lange die Schönheit unseres Faches erhalten können.

Herr Professor Katoh, vielen Dank für das Gespräch! 